\title{
A sum greater than its parts
}

\author{
Janice M. Leung ${ }^{1,2}$ and S.F. Paul Man ${ }^{1,2}$ \\ Affiliations: ${ }^{1}$ Centre for Heart Lung Innovation, University of British Columbia at St Paul's Hospital, Vancouver, \\ BC, Canada. 'Division of Respirology, University of British Columbia Dept of Medicine at St. Paul's Hospital, \\ Vancouver, BC, Canada.
}

Correspondence: S.F. Paul Man, Centre for Heart Lung Innovation, University of British Columbia at St Paul's Hospital, 1081 Burrard Street, Room 548, Vancouver, BC, V6Z 1Y6, Canada.

E-mail: pmanaprovidencehealth.bc.ca

0 @ERSpublications

Panels of biomarkers tailored for specific COPD phenotypes may represent our best hope as laboratory tests for COPD http://ow.ly/AjA9O

The pursuit of the perfect biomarker in chronic obstructive pulmonary disease (COPD) has been fraught with peril. The dream, of course, is of a single, accessible and inexpensive laboratory test that can more accurately diagnose COPD, define its severity, or fluctuate in accordance with disease progression and remission. In its ideal form, it would allow for earlier diagnosis of disease or earlier identification of the most severely affected patients, all with high sensitivity and specificity. Or it would properly indicate to a physician whether a particular COPD treatment has worked or failed. One only has to look to cardiology and its use of the troponin assay for the diagnosis of a myocardial infarction or to nephrology and its reliance on creatinine as a measure of renal function for such examples. If a similar biomarker is realised in COPD, it would significantly bolster what we can now predict through spirometry alone [1]. However, 20 years of painstaking research has led us no closer to this Holy Grail of biomarkers. To date, not one blood-based biomarker has reached meaningful clinical significance in COPD. Even the most promising candidates like C-reactive protein and fibrinogen, both of which have been shown to at least predict mortality in COPD, are nonspecific and lack the ability to distinguish manifestations of COPD from other inflammatory disorders $[2,3]$. Pneumoproteins like surfactant protein D and club cell secretory protein, which are primarily produced in the lung, could theoretically circumvent these limitations. However, only weak associations have been found between these biomarkers and important clinical benchmarks like exacerbation risk and lung function decline $[4,5]$. As a result, these tests remain the domain of research laboratories, intellectually interesting to ponder but largely irrelevant when considered within the context of actual patient management.

Where along the biomarker discovery journey have we gone wrong? Is it simply too preposterous to venture that a disease as complex and as incompletely understood as COPD could be boiled down to one mere laboratory test? Are we too narrow-minded in our approach to COPD, oversimplifying it as a single organ disease when in fact a scourge of damage permeates throughout the body? We now recognise that COPD is highly associated with a number of comorbidities including cardiovascular disease, osteoporosis, diabetes and metabolic syndrome [6-8]. Moreover, that simple, classic dichotomy between the "pink puffer" and the "blue bloater" and, more recently, between "emphysema" and "chronic bronchitis" fails to capture the multiplicity of COPD phenotypes. For example, frequent exacerbations, systemic inflammation and cachexia mark distinct subsets of COPD patients [9]. While the mechanisms explaining these unique phenotypes or the relationship between COPD and extrapulmonary comorbidities remain a mystery, clearly the notion of a single laboratory test capturing this diversity is hard to endorse. In this issue, SToLz et al. [10] have successfully rectified some of the past shortcomings of biomarker research by broadening the approach to biomarkers, which may have previously been too confined to allow for success. The authors have studied a panel of three biomarkers (adrenomedullin, arginine-vasopressin and atrial natriuretic

Received: July 092014 | Accepted after revision: July 202014

Conflict of interest: None declared.

Copyright @ERS 2014 
peptide) in patients with stable COPD, revealing that elevations in all three biomarkers are associated with a five-fold increased risk of death. Furthermore, the addition of this biomarker panel to the BODE (body mass index, airflow obstruction, dyspnoea, exercise capacity) index improved mortality predictions by $50 \%$.

Two important messages emerge from these data. First, the idea that a single biomarker can represent and encapsulate an entire disease process should now be put to rest. Single biomarkers are, by definition, onedimensional, grasping only a facet of what is otherwise a complicated disorder. By contrast, biomarker panels, over and above individual biomarkers, may possess greater discriminatory power in distinguishing worse prognoses from more stable clinical courses. This has now been demonstrated in several trials, including the cluster analysis of PINTO-PLATA et al. [11], which identified 19 key biomarkers that together corresponded well with exacerbation rates and BODE indices, and the work of CELLI et al. [12], which found that a panel of nine biomarkers significantly improved the $\mathrm{C}$ statistic for death over common clinical characteristics like age, BODE index and previous COPD hospitalisations, and also over the nine biomarkers themselves when considered in isolation. Similarly, transformations of multiple biomarkers have proven utility over single biomarkers, for example the ratio of fibronectin to C-reactive protein, which can better predict mortality outcomes compared with fibronectin and C-reactive protein alone [13]. The work of STOLZ et al. [10] echoes this pattern, emphasising the need to consider biomarkers collectively. Single elevations of adrenomedullin, arginine-vasopressin or atrial natriuretic peptide may not provide the clinician with much important information, but simultaneous elevations in all three are shown here to identify those patients at the greatest risk for death. The analysis of combinations of biomarkers will, in the future, probably provide the best predictive measures in COPD.

Secondly, one cannot help but note the distinct cardiovascular roles of the biomarkers studied. Arginine vasopressin is a critical hormone regulating fluid balance and vasoconstriction, while atrial natriuretic peptide is a vasodilating hormone produced by the heart to influence water, sodium and potassium balance. Adrenomedullin, although a contributing player in the cellular response to oxidative stress and hypoxia, is also a potent vasodilator. As such, these hormones are perhaps not the first biomarkers to come to mind when considering a lung predominant disorder like COPD. That they exhibit such strong signals reflecting COPD mortality, however, should not come as a surprise. The interplay between the heart and lung in COPD has long been established in the literature and deserves some attention. The prevalence of ischaemic heart disease and atrial fibrillation, for example, has been shown to be significantly higher in patients with COPD than in those without COPD [14]. Moreover, individuals with COPD carry a 2.27-fold increased risk of developing a myocardial infarction within the first 5 days of an exacerbation [15]. Elevations in B-type natriuretic peptide are also seen in acute exacerbations, even in the absence of overt heart failure [16]. Reasons for this overlap are speculative at this time, but proposed mechanisms include systemic inflammation originating from the cigarette smoke-exposed lungs that ultimately transits to the coronary vessels, driving endothelial activation. Sympathetic nervous system overdrive as a result of respiratory insufficiency and chronic hypoxia may also promote myocardial stress leading to heart failure.

What STOLz et al. [10] may have identified in this cohort with the particular panel of biomarkers studied is a unique and important COPD phenotype: this subset of COPD patients carry concurrent cardiovascular comorbidity to a high degree and as such, shoulder the greatest risk of death. Crucial clinical implications emerge from these data, including the need for physicians to rapidly identify (perhaps through the use of biomarkers, among other methods) and treat cardiovascular disease in COPD patients. Whether such interventions could alter the natural progression of the disease and extend survival rates remains to be answered. These data demonstrate that consideration of COPD as a single organ entity is an inherently flawed approach and may lead us to ignore the reality that COPD in fact produces microenvironments of damage well outside the lung. Successful future biomarker work in COPD may arise from recognising this diversity of effect, emphasising that we may well need to look beyond the lung to discover the most clinically applicable biomarkers in this complex disease. Panels of biomarkers tailored for specific COPD phenotypes may represent our best hope in solving this biomarker quandary.

\section{References}

1 Ashley F, Kannel WB, Sorlie PD, et al. Pulmonary function: relation to aging, cigarette habit, and mortality. Ann Intern Med 1975; 82: 739-745.

2 Duvoix A, Dickens J, Haq I, et al. Blood fibrinogen as a biomarker of chronic obstructive pulmonary disease. Thorax 2013; 68: 670-676.

3 Gan WQ, Man SF, Senthilselvan A, et al. Association between chronic obstructive pulmonary disease and systemic inflammation: a systematic review and a meta-analysis. Thorax 2004; 59: 574-580.

4 Lomas DA, Silverman EK, Edwards LD, et al. Serum surfactant protein D is steroid sensitive and associated with exacerbations of COPD. Eur Respir J 2009; 34: 95-102.

5 Park HY, Churg A, Wright JL, et al. Club cell protein 16 and disease progression in chronic obstructive pulmonary disease. Am J Respir Crit Care Med 2013; 188: 1413-1419. 
Divo M, Cote C, de Torres JP, et al. Comorbidities and risk of mortality in patients with chronic obstructive pulmonary disease. Am J Respir Crit Care Med 2012; 186: 155-161.

7 Jørgensen NR, Schwarz P, Holme I, et al. The prevalence of osteoporosis in patients with chronic obstructive pulmonary disease: a cross sectional study. Respir Med 2007; 101: 177-185.

8 Díez-Manglano J, Barquero-Romero J, Almagro P, et al. COPD patients with and without metabolic syndrome: clinical and functional differences. Intern Emerg Med 2014; 9: 419-425.

9 Vestbo J. COPD: definitions and phenotypes. Clin Chest Med 2014; 35: 1-6.

10 Stolz D, Meyer A, Rakic J, et al. Mortality risk prediction in COPD by a prognostic biomarker panel. Eur Respir J 2014; 44: 1557-1570.

11 Pinto-Plata V, Toso J, Lee K, et al. Profiling serum biomarkers in patients with COPD: associations with clinical parameters. Thorax 2007; 62: 595-601.

12 Celli BR, Locantore N, Yates J, et al. Inflammatory biomarkers improve clinical prediction of mortality in chronic obstructive pulmonary disease. Am J Respir Crit Care Med 2012; 185: 1065-1072.

13 Man SF, Xing L, Connett JE, et al. Circulating fibronectin to C-reactive protein ratio and mortality: a biomarker in COPD? Eur Respir J 2008; 32: 1451-1457.

14 Lange P, Mogelvang R, Marott JL, et al. Cardiovascular comorbidity in COPD: a study of the general population. COPD 2010; 7: 5-10

15 Donaldson GC, Hurst JR, Smith CJ, et al. Increased risk of myocardial infarction and stroke following exacerbation of COPD. Chest 2010; 137: 1091-1097.

16 Nishimura K, Nishimura T, Onishi K, et al. Changes in plasma levels of B-type natriuretic peptide with acute exacerbations of chronic obstructive pulmonary disease. Int J Chron Obstruct Pulmon Dis 2014; 9: 155-162. 